\title{
Secondary Colony Formation by Lactobacillus casei
}

\author{
By H. C. DE KLERK AND J. N. COETZEE \\ Department of Microbiology, University of Pretoria, Pretoria, South Africa
}

(Received 10 January 1962)

\section{SUMMARY}

In addition to $\mathbf{R}$ outgrowths, strains of Lactobacillus casei growing on a carbohydrate-free medium also formed smooth secondary colonies situated on the primary colonies. These secondary colonies arose after about 6 days of incubation and were of two types: when centrally situated they formed papillae; when near the margin of the mother colony they often spilt over and formed fan-shaped outgrowths adjacent to the parent colony. Cultures of several different species of Lactobacillus were examined; secondary colony formation was confined almost exclusively to strains of $L$. casei. The fan-shaped outgrowths were pure cultures of organisms possessing a shorter generation time than those comprising the mother colony. An inverse relationship existed on crowded and sparsely inoculated plates between numbers of papillae and fan-shaped outgrowths/colony observed. The distribution of colonies bearing different numbers of secondary colonies did not follow a Poisson distribution; the random origin of fast-growing variants could not be established. On subculture the fast-growing variants produced colonies with a slightly different morphology from that of the wild-types. This difference was stable and persisted through numerous subcultures. In the presence of small quantities of fermentable carbohydrates secondary colony formation was not observed and this possibly explains how the wild type competes successfully with fast-growing variants in natural environments.

\section{INTRODUCTION}

In a study of the S-R variation in Lactobacillus casei (de Klerk \& Coetzee, 1962) strains were plated on a carbohydrate-free medium. In addition to the $\mathbf{R}$ variants observed, secondary colonies in the form of smooth papillae often appeared on the surfaces of the colonies. A cursory examination of subcultures of the papillae on the same medium revealed colonies similar in appearance to the primary colony. Subsequently it was found that on crowded plates most of the smooth papillae which were situated peripherally continued to develop to form fan-shaped outgrowths which extended beyond the margins of the mother colony. The appearance of these outgrowths was remarkable (Pl. 1, fig. 1) and was encountered so frequently in subsequent work with the $\mathbf{R}$ variants that it was decided to investigate their nature.

\section{METHODS}

The media and the methods used for plating, testing biochemical reactions, making total counts of colony populations, and the phage and serological techniques were as described previously (de Klerk \& Coetzee, 1962). Incubation was always at $37^{\circ}$ unless otherwise stated. 
Organisms. Three strains of Lactobacillus casei var. casei $\left(\mathrm{C}_{9}, 300,316\right.$ used by de Klerk \& Coetzee, 1962) were examined in detail, and 15 other strains of $L$. casei var. casei, 10 strains of $L$. case $i$ var. rhamnosus, 5 of $L$. plantarum, 8 of L. acidophilus, 6 of $L$. salivarius, 10 of $L$. fermenti and 10 of $L$. brevis were examined for secondary colony formation. These strains had been isolated from human saliva and identified by the methods of Rogosa et al. (1953) and Wheater (1955).

Secondary colony formation. The effects of crowding on the development of papillae and fan-shaped outgrowths was studied by spreading $0.1 \mathrm{ml}$. of dilutions of overnight cultures grown in tomato glucose broth on to medium 1 agar (de Klerk \& Coetzee, 1962). After 8 days of incubation the distribution of secondary colonies in the form of papillae and fan-shaped outgrowths on parent colonies was determined in areas of $3 \mathrm{~cm} .^{2}$. The effect of the depth of medium on the incidence of the two types of smooth outgrowth was investigated by using different volumes of agar in similar Petri dishes. The effect of amino acids and vitamins on secondary colony formation was tested by incorporating vitamin-free pancreatic casein digest (Nutritional Biochemicals Corporation, Cleveland, Ohio, U.S.A.) at 10 g./l. into medium I. Ascorbic acid, nicotinic acid, thiamine hydrochloride, calcium D-pantothenate, riboflavin, $p$-aminobenzoic acid, DL-biotin, folic acid and vitamin $\mathrm{B}_{12}$ (all obtained from Nutritional Biochemicals Corporation) were incorporated in medium I in the quantities and by the methods as used by Rogosa, Franklin \& Perry (1961). The effect of addition of fermentable and non-fermentable carbohydrates was investigated as previously described (de Klerk \& Coetzee, 1962). The carbohydrates used were glucose, cellobiose, rhamnose and melibiose.

Colony diameters. Suspensions were made of samples of organisms from fan-shaped outgrowths and from areas of the mother colonies which were devoid of secondary colonies. Suitable dilutions to yield about $\mathbf{5 0}$ colonies/plate were seeded on to medium I agar. Colonies derived from these two sources will subsequently be referred to as $\mathbf{F}$ variants and wild-types, respectively. The diameters of fifty wellseparated colonies were measured after incubation for $48 \mathrm{hr}$. This was done with a micrometer eyepiece at $\times \mathbf{4 1 \cdot 2 5}$ magnification.

Generation times. Overnight cultures of single $\mathbf{F}$ variant and wild-type colonies in $5 \mathrm{ml}$. liquid medium I were transferred into $100 \mathrm{ml}$. quantities of fresh prewarmed liquid medium I. These cultures were maintained at $37^{\circ}$ and at suitable intervals samples removed. The optical densities of these samples were read with a Zeiss spectrophotometer at $660 \mathrm{~m} \mu$. Total counts on the samples, suitably diluted in cold distilled water, were done with a Petroff-Hauser counting chamber and a phase-contrast microscope. Dilutions of the samples were also plated for viable counts.

Changes in $\boldsymbol{p H}$ value of cultures in medium $I$. Cultures of $\mathbf{F}$ variants and wild strains were grown in $250 \mathrm{ml}$. liquid medium I for $170 \mathrm{hr}$., sampled at intervals, and readings of $\mathrm{pH}$ values of the samples made with a Beckman $\mathrm{pH}$ meter.

\section{RESULTS}

Colonies of all strains of Lactobacillus casei var. casei and $L$. casei var. rhamnosus and one strain each of $L$. fermenti and $L$. brevis gave secondary colonies on medium I agar which appeared as papillae anywhere on their surfaces or margins. These out- 
growths generally appeared between the fifth and seventh days of incubation. No secondary colony formation was ever seen on colonies of the other species examined. The number of secondary colonies formed by the single strains of $L$. fermenti and $L$. brevis mentioned was negligible in comparison with their numbers on colonies of the strains of $L$. casei. Secondary colony formation on this medium thus appeared to be a feature of strains of the latter species. The secondary colonies varied in size and were smooth and glistening (Pl. 1, figs. 2, 3). When situated centrally they soon stopped growing; but those at the periphery often spilt over the margin of the mother colony. Once in contact with the medium, they grew rapidly into large fan-shaped outgrowths, retaining their smooth glistening surfaces and entire edges (Pl. 1, fig. 4). Adjacent outgrowths did not coalesce (Pl. 1, fig. 5) as $\mathrm{R}$ variants tend to do (de Klerk \& Coetzee, 1962). Forty-eight hr. colonies of organisms from the mother colony (wild-type) and the fan-shaped outgrowths (F variants) on medium $I$, were smooth, but differed in that the latter were larger and slightly more raised and opaque (Pl. 1, fig. 6). This morphological difference persisted through numerous subcultures. Subcultures from the fan-shaped outgrowths were homogeneous and yielded only $\mathbf{F}$ variants. Subcultures from papillae situated centrally usually yielded mixtures of two types of colony, corresponding to the descriptions of $F$ variant and wild type given above. On other occasions only one or other of the colony types was recognized in subcultures from papillae. It was not possible to tell the composition of a papilla from its appearance. Microscopically, organisms from the mother colony and fan-shaped outgrowths or from subcultures of these on agar or in fluid media possessed identical morphologies. The organisms were arranged singly and in chains of 2 to 6 bacilli. The differences in size of the two colony types on medium I agar was verified by direct measurement: the mean diameter of colonies of the wildtype strain 300 was $0.46 \mathrm{~mm}$.; the mean diameter of the $\mathrm{F}$ variant of this strain was $0.55 \mathrm{~mm}$. Wild-type and $\mathrm{F}$ variant colonies of the other two strains showed similar differences. This difference in diameter was paralleled by results of total and viable counts. These results are presented in Table 1.

The tendency of these organisms to grow in chains of different lengths is one of the factors responsible for the viable counts being lower than the total counts. On further incubation of $\mathbf{F}$ variant and wild-type colonies on medium $\mathbf{I}$ agar, $\mathbf{R}$ outgrowths often appeared on both types of colony, but secondary colony formation was detected only on wild-type colonies. $R$ outgrowths were occasionally seen on fan-shaped outgrowths (Pl. 1, fig. 7) and frequently a wild-type colony bore both fan-shaped outgrowths and $R$ outgrowths (Pl. 1, fig. 8). No change in the incidence of secondary colony formation was detected on medium I supplemented with amino acids and/or vitamins. As with the $\mathrm{R}$ variants of $L$. casei (de Klerk \& Coetzee, 1962) neither form of secondary colony appeared on medium $I$ agar supplemented with glucose or cellobiose in excess of $0.5 \%(w / v)$. Addition of rhamnose or melibiose (not normally fermented by $L$. casei) did not prevent secondary colony formation.

The morphological differences between the two types of colonies noted on medium I agar disappeared when these were subcultured on tomato glucose agar. Forty-eight hr. colonies of wild-types and $\mathbf{F}$ variants were circular, white, opaque, about $2 \mathrm{~mm}$. in diameter, dome-shaped with an entire edge, with the surface very finely granular and glistening. The $F$ variant of strain 300 differed from the $R$ variant of this strain in that colonies of the $R$ variant retained their rough appearance 
on tomato glucose agar (de Klerk \& Coetzee, 1962). The effect of inoculum-size on the formation of papillae and fan-shaped outgrowths by strain $C_{9}$ is shown in Table 2.

\title{
Table 1. Total and viable counts of organisms in $48 \mathrm{hr}$.-colonies of wild strains and $\boldsymbol{F}$ variants of Lactobacillus casei
}

\begin{abstract}
Six whole colonies of each strain were cut out with the underlying agar and thoroughly emulsified in separate $0.25 \mathrm{ml}$. volumes of distilled water. The organisms present in samples were counted in a Petroff-Hauser chamber with a phase-contrast microscope. Suitable dilutions were plated on tomato glucose agar for viable counts. Colonies were enumerated after incubation for $48 \mathrm{hr}$. at $37^{\circ}$.
\end{abstract}

\begin{tabular}{lcc}
\multicolumn{1}{c}{ Strain } & $\overbrace{\begin{array}{c}\text { Viable count } \\
\text { (millions) }\end{array}}^{\begin{array}{c}\text { Mean populations of six } \\
\mathbf{4 8} \text { hr. colonies }\end{array}}$ & $\begin{array}{c}\text { Total count } \\
\text { (millions) }\end{array}$ \\
C $_{9}$ wild & $\mathbf{1 \cdot 2}$ & $4 \cdot 3$ \\
C $_{9}$ variant $F$ & $\mathbf{2 \cdot 9}$ & $\mathbf{8 \cdot 0}$ \\
300 wild & $1 \cdot 0$ & $\mathbf{3 \cdot 6}$ \\
300 F variant & $\mathbf{2 \cdot 5}$ & $\mathbf{6 \cdot 0}$ \\
316 wild & $\mathbf{3 \cdot 3}$ & $\mathbf{5 \cdot 0}$ \\
316 F variant & $\mathbf{4 \cdot 4}$ & $\mathbf{7 \cdot 5}$
\end{tabular}

Table 2. The effect of crowding on the development of papillae and fan-shaped outgrowths of Lactobacillus casei strain $\mathbf{C}_{9}$

Samples $(0.1 \mathrm{ml}$.) of 4 different dilutions of overnight growth of cultures in tomato glucose broth were spread on medium $I$ in similar Petri dishes. These were incubated at $37^{\circ}$ in an atmosphere of carbon dioxide for 8 days. Counts were done on 8 areas of $3 \mathrm{~cm} .{ }^{2}$ for each dilution.

\begin{tabular}{|c|c|c|c|}
\hline \multirow[b]{2}{*}{ Dilution } & \multirow[b]{2}{*}{$\begin{array}{c}\text { No. of } \\
\text { colonies } / 72 \mathrm{~cm} .^{2}\end{array}$} & \multicolumn{2}{|c|}{ Mean no. of secondary colonies } \\
\hline & & $\begin{array}{l}\text { Fan-shaped out- } \\
\text { growths/colony }\end{array}$ & Papillae/colony \\
\hline $1 \times 10^{-5}$ & 1804 & $0 \cdot 44$ & 1.95 \\
\hline $5 \times 10^{-6}$ & 488 & 0.28 & $3 \cdot 24$ \\
\hline $1 \times 10^{-6}$ & 160 & 0.04 & $3 \cdot 49$ \\
\hline $5 \times 10^{-7}$ & 56 & 0 & 5.72 \\
\hline
\end{tabular}

The incidence of fan-shaped outgrowths/colony bore a direct relationship to the number of colonies/unit area. The number of papillae/colony on the other hand had an inverse relationship. Experiments with strains 300 and 316 yielded similar results. Distribution of colonies bearing different numbers of fan-shaped outgrowths or papillae, or the sum of the latter two did not approach Poisson distributions at any of the degrees of crowding used in the above experiments. Differences in the thickness of the layer of medium had no effect on secondary colony formation. F variants and wild-types had the same biochemical reactions and growth temperatures.

The results of agglutination and agglutinin absorption tests with sera prepared against wild-types and $F$ variants of strains $C_{9}$ and 316 are shown in Table 3.

Unlike the $\mathbf{R}$ mutants of Lactobacillus casei previously described (de Klerk \& Coetzee, 1962) $\mathrm{F}$ variants of strains $\mathrm{C}_{9}, 316$ and 300 were found to be antigenically identical to the corresponding wild-types. L. casei strains 300 and 316 are susceptible 
to lytic phages 300 and 316 respectively (Coetzee, de Klerk \& Sacks, 1960). These phages had efficiencies of plating on the $F$ variants of their hosts identical to those on the wild strains. $R$ variants of strain 316 did not adsorb phage 316 (de Klerk \& Coetzee, 1962). No phage active on strain $C_{9}$ was available. The changes in $\mathrm{pH}$ value in medium $I$ liquid cultures of wild-types and $F$ variants were identical and minimal; during incubation for $170 \mathrm{hr}$. there was a gradual change from $\mathrm{pH} \mathrm{6.35}$ to 6.7. Measurement of the optical density of cultures growing in liquid medium $I$ are

Table 3. Results of agglutination and agglutinin absorption tests on antisera prepared against the wild-types and the $F$ variants of Lactobacillus casei strains $C_{9}$ and 316

\begin{tabular}{|c|c|c|c|}
\hline Antiserum & Serum absorbed with & Antigen & Titre \\
\hline $\mathrm{C}_{9}$ (wild-type) & $\begin{array}{c}-\overline{-} \\
C_{\vartheta} \text { (F variant) } \\
C_{9} \text { (F variant) }\end{array}$ & $\begin{array}{l}\mathrm{C}_{9} \text { (wild-type) } \\
\mathrm{C}_{9} \text { (F variant) } \\
\mathrm{C}_{9} \text { (F variant) } \\
\mathrm{C}_{9} \text { (wild-type) }\end{array}$ & $\begin{aligned} & 1 / 1024 \\
& 1 / 1024 \\
< & 1 / 8 \\
< & 1 / 8\end{aligned}$ \\
\hline$C_{9}(F$ variant $)$ & $\begin{array}{c}\overline{-} \\
\mathrm{C}_{9} \text { (wild-type) } \\
\mathrm{C}_{9} \text { (wild-type) }\end{array}$ & $\begin{array}{l}C_{9} \text { (F variant) } \\
C_{9} \text { (wild-type) } \\
C_{9} \text { (wild-type) } \\
C_{9} \text { (F variant) }\end{array}$ & $\begin{aligned} & 1 / 1024 \\
& 1 / 512 \\
< & 1 / 16 \\
< & 1 / 16\end{aligned}$ \\
\hline 316 (wild-type) & $\begin{array}{c}\overline{-} \\
316 \text { (F variant) } \\
316 \text { (F variant) }\end{array}$ & $\begin{array}{l}316 \text { (wild-type) } \\
316 \text { (F variant) } \\
316 \text { (F variant) } \\
316 \text { (wild-type) }\end{array}$ & $\begin{aligned} & 1 / 2048 \\
& 1 / 2048 \\
< & 1 / 16 \\
< & 1 / 16\end{aligned}$ \\
\hline 316 (F variant) & $\begin{array}{l}\overline{-} \\
316 \text { (wild-type) } \\
316 \text { (wild-type) }\end{array}$ & $\begin{array}{l}316 \text { (F variant) } \\
316 \text { (wild-type) } \\
316 \text { (wild-type) } \\
316 \text { (F variant) }\end{array}$ & $\begin{aligned} & 1 / 1024 \\
& 1 / 1024 \\
< & 1 / 8 \\
< & 1 / 8\end{aligned}$ \\
\hline
\end{tabular}

Controls with $0.8 \%(\mathrm{w} / \mathrm{v}) \mathrm{NaCl}$ solution were satisfactory.

presented in Fig. 1. The slopes of these lines show that the F variants of $L$. case $i$ grew more rapidly than the wild strains under the conditions of the experiment. Generation times of wild-types and $F$ variants calculated from viable counts during the exponential period of growth always showed those of the $F$ variant to be the shorter. The actual times obtained with any pair of strains were, however, so nonreproducible that this method of obtaining the absolute generation times was soon abandoned. This irregular behaviour was attributed to the fact that $L$. casei grew in chains of different lengths. Generation times of the wild-type and F variant of strain 300, calculated from total counts in six different experiments, varied from 173 to $185 \mathrm{~min}$. for the wild-type and from 147 to $154 \mathrm{~min}$. for the $\mathrm{F}$ variant. These results are considered to be reliable since organisms in chains could be enumerated individually and deaths during the period of the experiment (usually about $10 \mathrm{hr}$.) would be minimal. Experiments with wild-types and $F$ variants of strains $\mathbf{C}_{9}$, and 316 yielded similar results. These results confirm the indications obtained above that $\mathbf{F}$ variants have shorter generation times than wild-types. 
Subcultures were made from centrally situated papillae of colonies of the three strains examined. A limited number of $\mathbf{F}$ variant-like colonies and wild-type-like colonies so obtained were examined by all the above methods. Organisms comprising the two types of colony were found to possess properties identical with those in $\mathrm{F}$ variant and wild-type colonies, respectively.

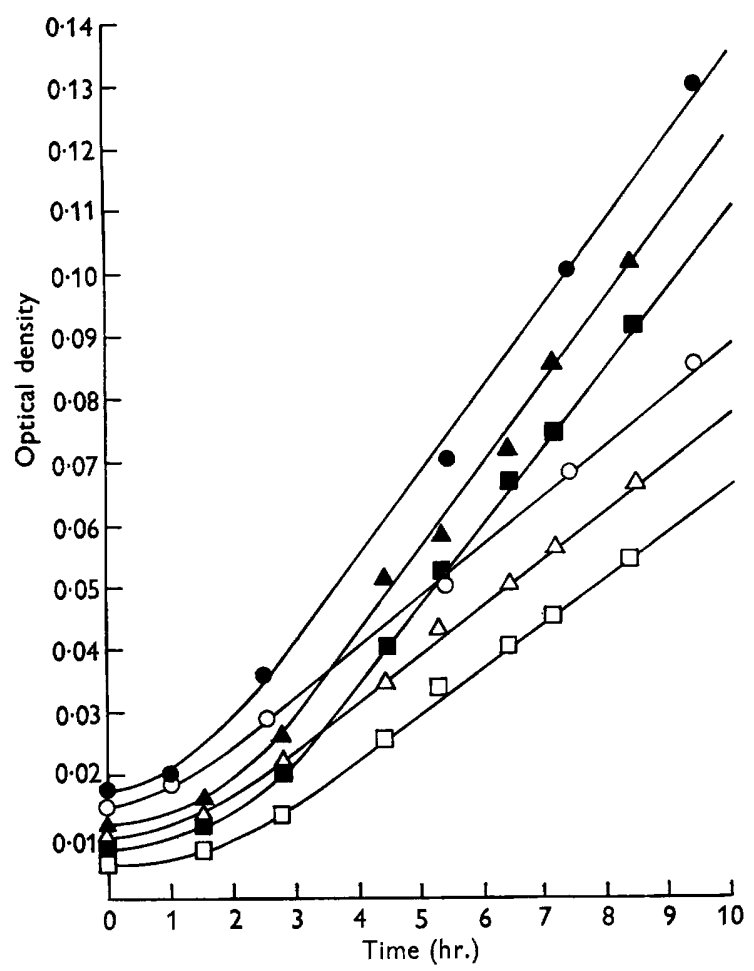

Fig. 1. Photometric determinations of growth of wild-types and $F$ variants of strains $C_{9}, 300$ and 316. Overnight cultures of wild-type and $F$ variant colonies in $6 \mathrm{ml}$. of medium I broth were transferred into $100 \mathrm{ml}$. volumes of fresh pre-warmed broth and maintained at $37^{\circ}$ for $10 \mathrm{hr}$. At intervals samples were removed and optical densities read on a Zeiss spectrophotometer at $660 \mathrm{~m} \mu$. Strain 300 wild-type, $\bigcirc$; strain 300 F variant, $\odot$. Strain $C_{9}$ wild-type, $\Delta ;$ strain $C_{9} F$ variant, $\Delta$. Strain 316 wild-type, $\square$; strain $316 \mathrm{~F}$ variant, $\mathrm{E}$.

\section{DISCUSSION}

It is reasonable to suppose that organisms able to grow out above their relations in a colony must possess some selective advantage. This has been proved in many instances, see for example, the paper by Ryan, Schwartz \& Fried (1955). These workers described the development of papillae arising on colonies of a histidineless strain of Escherichia coli, growing in the presence of subminimal quantities of histidine. The selective advantage enjoyed by the mutant organisms comprising these papillae was found to be due to the fact that they were histidine independent. Shah \& Iyer (1960) showed that organisms in secondary colonies of Bacillus subtilis were favoured by their greater ability to neutralize the acids initially produced by fermentation in colonies on a medium containing glucose and peptone. The Lacto- 
bacillus casei $\mathbf{F}$ variants encountered in the present work differed from the wildtypes in that colonies of the former were larger, more opaque and contained more bacilli than the latter when growing on medium I. These differences may be associated with the observation that the $F$ variants had relatively shorter generation times than the wild-types under the conditions of study. Absolute generation times could only be determined by means of total counts. The times obtained were much longer than those of $L$. casei and other lactobacilli determined under optimal conditions by a variety of methods (Mason, 1935). This was probably due to the poor growth-supporting qualities of medium I. It is interesting to note that $L$. casei has the shortest generation time of all the species of lactobacilli listed by Mason (1935). It might have been anticipated that fast-growing mutants of other species of lactobacilli which have longer generation times would possess a greater selective advantage and be encountered more frequently, yet secondary colony formation was practically confined to strains of $L$. casei var. casei and $L$. casei var. rhamnosus. Fast-growing variants of $\boldsymbol{E}$. coli detected in continuous cultures have been reported by Novick \& Szilard (1950) and by Moser (1955). In the present work the fan-shaped outgrowths were pure cultures of $\mathbf{F}$ variants but uncertainty still remains regarding the nature of many papillae. Most papillae apparently consisted of mixtures of $F$ variant and wild-type organisms. Some consisted of pure cultures of $F$ variantproducing organisms, but subculture of many papillae produced only wild-type colonies. It may be argued that a papilla is a dynamic structure and that it might, at a particular stage of its development, still be contaminated with parent organisms. Despite the limited means of identification available this could hardly explain the many instances where no $\mathbf{F}$ variant-like colonies were detected in subcultures of well-developed papillae. Loss of viability of $\mathbf{F}$ variants in papillae with survival of contaminating wild-type organisms was considered as an explanation of the latter finding. This appears to be unlikely. First, because there were no indications that F variant organisms tended to die off more rapidly than the wild-type, and secondly because $F$ variants were often present in other papillae on the same colony. The fact that all secondary colonies failed to appear on media containing some carbohydrate is no argument for the basic homogeneity of these colonies. $\mathbf{R}$ mutants (de Klerk \& Coetzee, 1962) also lose their selective advantage under these circumstances. Another argument in favour of the basic heterogeneity of secondary outgrowths is the fact that the distributions of colonies bearing homogeneous outgrowths and papillae often follow a Poisson distribution (Ryan et al. 1955; Coetzee, 1959; Coetzee \& Sacks, $1960 a, b$; de Klerk \& Coetzee, 1962). The fact that the distribution of colonies of $L$. case $i$ bearing varying numbers of secondary colonies did not follow such a distribution is another factor against the inherent identity of all these structures. However, an inverse relationship existed between numbers of papillae and fan-shaped outgrowths/colony on crowded and on sparsely inoculated plates. This might be explained on the basis that on crowded plates the colonies were smaller and secondary colonies which arose in the form of papillae thus stood a greater chance of spilling over the margins of colonies and developing into fan-shaped outgrowths. This could suggest that many papillae and fan-shaped outgrowths were of the same composition. Because other undetected phenotypic differences (and possible selective advantages) might be involved it was decided not to couple the single manifestation of fast growth with secondary colony formation. For 
reasons mentioned above it has not been possible to obtain proof of the random origin of the $\mathrm{F}$ variants and to determine the mutation rates involved. The fanshaped outgrowths, however, may be regarded as fast-growing variants of $L$. casei which possess a selective advantage under the austere conditions prevailing in growth on medium I. These variants maintained their characteristics through numerous platings and were stable in the environment tested. Like the $\mathbf{R}$ mutants, however (de Klerk \& Coetzee, 1962), they lost their selective advantage and were not observed in the presence of small amounts of fermentable carbohydrates. This is a possible explanation why the wild-type maintains itself as the modal form.

This work is submitted with permission of Professor C. L. de Jager, honorary director of the Nutrition and Dental Health Research Group of the South African Council for Scientific and Industrial Research. Acknowledgement is made to Miss I. R. Leemann and Mrs C. S. Steyn for technical assistance rendered.

\section{REFERENCES}

Coetzee, J. N. (1959). The late lactose-fermenting property of Shigella sonnei. S. Afr. J. Lab. clin. Med. 5, 170.

Coetzee, J. N., DE KLeRK, H. C. \& SACKs, T. G. (1960). Host range of lactobacillus bacteriophages. Nature, Lond. 187, 348.

Coetzee, J. N. \& SACKs, T. G. (1960a). The late sucrose fermenting property of Proteus mirabilis. S. Afr. J. Lab. clin. Med. 6, 49.

Coetzee, J. N. \& Sacks, T. G. (1960b). Morphological variants of Proteus hauseri. J. gen. Microbiol. 23, 209.

DE Kuerk, H. C. \& Coetzee, J. N. (1962). The S-R variation in Lactobacillus casei. J. gen. Microbiol. 27, 155.

MAson, M. M. (1935). A comparison of maximal growth rates of various bacteria under optimal conditions. J. Bact. 29, 103.

Moser, H. (1955). Carnegie Inst. (Wash.), Yearbook, no. 54, p. 231.

Novick, A. \& Szillard, L. (1950). Experiments with the chemostat on spontaneous mutations of bacteria. Proc. nat. Acad. Sci., Wash. 36, 708.

Rogosa, M., Frankitin, J. G. \& Perry, K. D. (1961). Correlation of the vitamin requirements with cultural and chemical characters of Lactobacillus spp. J. gen. Microbiol. 25, 473.

Rogosa, M., Wiseman, R. F., Mitchell, J. A., Disraely, M. N. \& Beaman, A. J. (1953). Species differentiation of oral lactobacilli from man including descriptions of Lactobacillus salivarius nov.spec. and Lactobacillus cellobiosus nov.spec. J. Bact. 65, 681 .

Ryan, F. J., Scrwartz, M. \& Fried, P. (1955). The direct enumeration of spontaneous and induced mutations in bacteria. J. Bact. 69, 552.

ShAH, K. K. \& IYer, V. N. (1960). Secondary colony formation by Bacillus subtilis on eosine methylene-blue agar. J. Bact. 81, 887.

WheATER, D. M. (1955). The characteristics of Lactobacillus acidophilus and Lactobacillus bulgaricus. J. gen. Microbiol. 12, 123.

\section{EXPLANATION OF PLATE}

Colonial morphology of Lactobacillus casei strains on medium I agar.

Fig. 1. Crowded 6-day colonies of strain $C_{9}$ showing four fan-shaped outgrowths and numerous minute papillae. An $R$ outgrowth is seen on one of the fan-shaped outgrowths. $\times 25$.

Fig. 2. 7-day colonies of strain 300 showing numerous smooth papillae of different sizes. An $R$ outgrowth and fan-shaped outgrowth are also present. $\times 40$.

Fig. 3. 4-day colonies of strain 300 showing smooth papillae on their surfaces and at least two early fan-shaped outgrowths at the margins. $\times 25$. 
Journal of General Microbiology, Vol. 29, No. 2

Plate 1
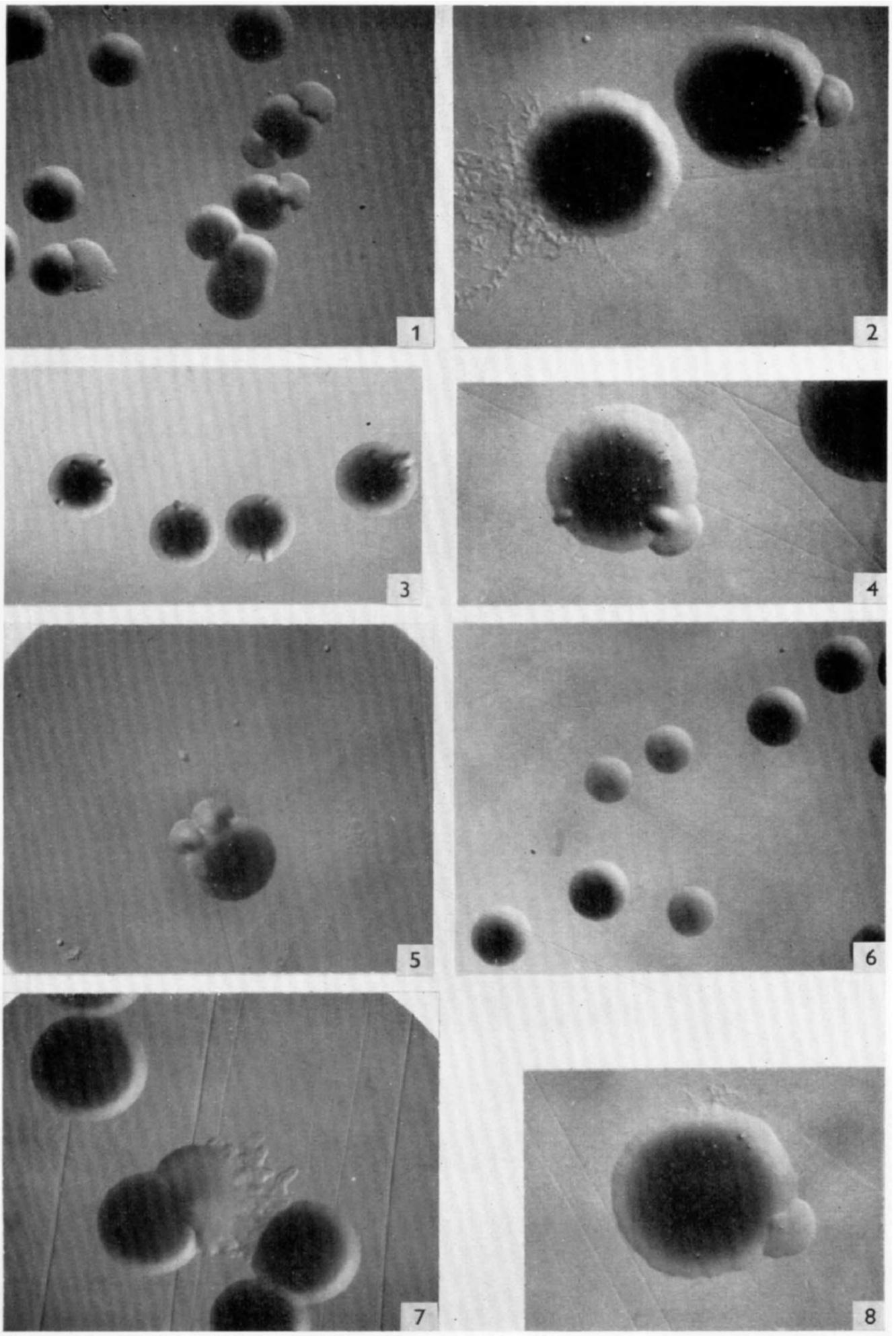

H. C. DE KLERK AN J. N. COETZEE

(Facing p. 298) 

Fig. 4. 6-day colony of strain 316 showing numerous smooth papillae and one early and one fully developed fan-shaped outgrowth. $\times 40$.

Fig. 5. 6-day colony of strain $C_{9}$ showing sharp demarcation between adjacent fan-shaped outgrowths. $\times 25$.

Fig. 6. 2-day colonies of the wild-type and $F$ variant of strain 316. F variant colonies are clearly distinguishable by their larger diameter and greater density. $\times 25$.

Fig. 7. A fan-shaped outgrowth on a 6-day colony of strain $C_{9}$ showing the development of an $R$ outgrowth from its margin. $\times 40$.

Fig. 8. 8-day colony of strain 300 with a tan-shaped and $R$ outgrowth on its margin. Numerous papillae are present on the surface. $\times 40$. 
